\title{
Association between metabolic syndrome and vascular endothelium dysfunction in children and adolescents
}

\author{
Y. Wei, G.L. Liu, J.Y. Yang, R.X. Zheng, L.H. Jiang, Y.P. Li and F.F. Gao \\ Department of Pediatrics, General Hospital of Tianjin Medical University, \\ Tianjin, China \\ Corresponding author: G.L. Liu \\ E-mail: liugeli008@sina.com
}

Genet. Mol. Res. 13 (4): 8671-8678 (2014)

Received July 11, 2013

Accepted December 5, 2013

Published October 27, 2014

DOI http://dx.doi.org/10.4238/2014.October.27.7

\begin{abstract}
We aimed at investigating the association between metabolic syndrome (MS) and vascular endothelial cell dysfunction (ECD) in children and adolescents. Sixty children (30 obese children and 30 children with MS) were included in this retrospective analysis. Thirty healthy subjects were randomly selected as the control group. A series of indices/biomarkers known to be related to MS/ECD were determined using ELISA. Correlations between the variables measured were analyzed. Compared with the control group, PAI-1, vWF, VEcad, TM, and VEGF were significantly increased in the MS group (P $<0.05)$. Adolescents in the obese group had significantly increased levels of serum PAI-1, VE-cad, TM, and VEGF as compared with the control group $(\mathrm{P}<0.05)$. Further, vWF in the obese and control groups did not differ significantly $(\mathrm{P}=0.556)$. Our results suggest that ECD is correlated with MS in children and adolescents. Pathophysiological changes of the vascular endothelium may exist in obese children who have yet to develope MS. PAI-1, vWF, VE-cad, TM, and VEGF could be used as biomarkers for predicting ECD. ECD that develops in patients with MS may be associated with obesity, elevated blood lipid,
\end{abstract}


elevated blood glucose, and higher blood pressure.

Key words: Metabolic syndrome; Vascular endothelium dysfunction; Plasminogen activator inhibitor-1; von Willebrand factor; Vascular endothelial growth factor

\section{INTRODUCTION}

Metabolic syndrome (MS) has been shown to be related to certain genes. Gene-environment interactions can cause MS, which negatively affects the nervous system and endocrine regulation, causing fluctuations in glucose metabolism, abnormal lipid metabolism, and eventually leading to many clinical diseases including cardiovascular disease (CVD) and type 2 diabetes mellitus (T2DM) (Lim et al., 2004).

Vascular endothelial dysfunction clinically manifests as a series of reactions, including decreased endothelium-dependent vasorelaxation, increased vascular permeability, and the abscission and reparation of endothelial cells. These are the initial signs of CVD and T2DM. In recent years, the prevalence of these chronic, metabolism-related disorders has increased among young people in China. In this study, we aim to investigate the relationship between MS and vascular endothelial cell dysfunction (ECD) in adolescents and children.

\section{MATERIAL AND METHODS}

\section{Subjects}

A total of 60 subjects presented to General Hospital of Tianjin Medical University from February 2011 to February 2012 were included in this study. Additionally, 30 healthy individuals were randomly selected as control. All the subjects signed the informed consents. This study was approved by the Ethics Committee of General Hospital of Tianjin Medical University.

The body mass index (BMI) reference norm for screening overweight and obesity in Chinese children and adolescents, as defined by the Chinese obesity task force of the International Committee of Life Sciences in 2004, was chosen as the diagnostic criteria of overweight or obesity. Cases of morbid obesity and obesity syndrome caused by other diseases other than MS were excluded (Expert Panel on Detection Evaluation aToHBCiA, 2001).

The MS diagnostic criteria for children and adolescents, proposed by the International Diabetes Federation (IDF) in 2007, were used to define cases of MS in this study (Zimmet et al., 2007).

\section{Measurement of physical parameters}

The height $(\mathrm{H})$ and weight $(\mathrm{W})$, in $\mathrm{cm}$ and $\mathrm{kg}$, respectively, of fasting subjects wearing unlined garments and no shoes were measured with an accuracy of $0.1 \mathrm{~kg}$ and $0.1 \mathrm{~cm}$, respectively.

BMI was defined as body weight $(\mathrm{kg})$ divided by the square of the height $\left(\mathrm{m}^{2}\right)$, in accordance with the WHO recommendations. To measure waist and hip circumference, children were instructed to stand vertically and breathe steadily. An inelastic tape measure was placed on the midpoint of the upper edge of the hip bone in the right axillary line and the lower edge of the twelfth rib (usually the slimmest parts of the waist) and horizontally wrapped around the abdo- 
men close to the skin, but without compression. The length value was then read and recorded. Hip circumference (HC) was measured as the length of both sides of the greater trochanter, taking the pubic symphysis as the front and the most prominent part of the buttocks as the back.

Blood pressure was measured using a mercury sphygmomanometer. Before blood pressure was measured, all children were instructed to sit quietly for 5-10 min. Following this, brachial arterial blood pressure including systolic blood pressure (SBP) and diastolic blood pressure (DBP) was measured on their right upper arm. Continuous measurement times were presented as averages. All data were measured twice continuously, and the average values were used.

\section{Detection of indicators of glucose and lipid metabolism}

All subjects fasted for over $8 \mathrm{~h}$ before being tested. In the morning, fasting blood glucose (FBG) and fasting insulin (FIN) levels in the venous blood of participants were determined, from which insulin resistance index (IR) was calculated. Serum total cholesterol (TC), serum triglycerides (TG), low-density lipoprotein (LDL) cholesterol, high-density lipoprotein (HDL), and cholesterol were also determined. Blood glucose was measured using the glucose oxidase method, insulin levels were measured using radioimmunoassay, and lipids were measured using the enzymatic method.

\section{Measurement of serum plasminogen activator inhibitor-1 (PAI-1), von Willebrand Factor (vWF), vascular endothelial cadherin (VE-cad), thrombomodulin (TM), and vascular endothelial growth factor (VEGF)}

Venous blood serum concentrations of PAI-1, vWF, VE-cad, TM, and VEGF were measured using enzyme-linked immunosorbent assay (ELISA), following $8 \mathrm{~h}$ of fasting.

\section{Statistical analysis}

Statistical analysis was performed using SPSS 17.0 (SPSS Inc., Chicago, IL, USA). All data are reported as means \pm standard deviation. One-way ANOVA analysis was performed for inter-group comparisons. The correlation between each indicator and serum concentrations of PAI1, vWF, VE-cadherin, TM, and VEGF were calculated using the Pearson's rank correlation coefficient method, with rs as the correlation coefficient, where an $r_{\mathrm{s}}$ of $<0$ was considered to be a negative correlation, and an $r_{\mathrm{s}}$ value of $>0$ was considered to be a positive correlation. The statistical significance level was $\alpha=0.05$, and $\mathrm{P}$ values of $<0.05$ were considered to be statistically significant.

\section{RESULTS}

\section{Concentrations of PAI-1, vWF, VE-cad, TM, and VEGF}

In the MS group, concentrations of PAI-1, vWF, VE-cad, TM, and VEGF showed significant increase compared to those in the control group $(\mathrm{P}<0.05$, Table 1$)$. In the obesity group, a significant increase was noted in the concentrations of PAI-1, VE-cad, TM, and VEGF compared with the control group $(\mathrm{P}<0.05)$. Compared with the obesity group, remarkable increase was noted in the concentration of vWF and VE-cad in the MS group $(\mathrm{P}<0.05)$. 
Table 1. Levels of PAI-1, vWF, VE-cad, TM, and VEGF.

\begin{tabular}{lcccccc}
\hline Group & $\mathrm{N}$ & $\mathrm{PAI}-1(\mu / \mathrm{L})$ & $\mathrm{vWF}(\mu / \mathrm{L})$ & $\mathrm{VE}-\mathrm{cad}(\mu \mathrm{g} / \mathrm{mL})$ & $\mathrm{TM}(\mathrm{ng} / \mathrm{mL})$ & $\mathrm{VEGF}(\mathrm{pg} / \mathrm{mL})$ \\
\hline MS group (1) & 30 & $106.05 \pm 5.11$ & $106.82 \pm 21.67$ & $3.50 \pm 1.07$ & $1.75 \pm 0.68$ & $86.28 \pm 38.08$ \\
Obesity group (2) & 30 & $105.53 \pm 2.02$ & $94.37 \pm 20.79$ & $2.50 \pm 0.88$ & $1.64 \pm 0.62$ & $79.56 \pm 24.23$ \\
Control group (3) & 30 & $102.35 \pm 4.43$ & $91.32 \pm 17.40$ & $1.81 \pm 1.04$ & $1.120 \pm 0.61$ & $60.75 \pm 29.98$ \\
$F$ & & 7.246 & 5.044 & 23.411 & 8.772 & 5.690 \\
P(1):(2) & & 0.623 & $0.018^{*}$ & $0.013^{*}$ & 0.515 & 0.212 \\
P(1):(3) & $0.001^{*}$ & $0.004^{*}$ & $0.013^{*}$ & $0.000^{*}$ & $0.002^{*}$ \\
P(2):(3) & $0.003^{*}$ & 0.556 & $0.000^{*}$ & $0.004^{*}$ & $0.018^{*}$
\end{tabular}

$* \mathrm{P}<0.005$ indicates significant increase. PAI- $1=$ serum plasminogen activator inhibitor-1; vWF $=$ von Willebrand factor; VE-cad = vascular endothelial cadherin; $\mathrm{TM}=$ thrombomodulin; VEGF = vascular endothelial growth factor; MS = metabolic syndrome.

\section{Correlations of serum PAI-1, vWF, VE-cad, TM, and VEGF with BMI, blood fat, blood glucose, and insulin concentration}

Serum PAI-1 was positively associated with body weight, BMI, waist circumference (WC), waist/hip ratio (WHR), TC, TG, LDL, FBG, FIN, SBP, and DBP $(\mathrm{P}<0.05)$. Additionally, PAI-1 was negatively associated with HDL $(\mathrm{P}<0.05)$. No correlation was found between $\mathrm{HC}$ and IR $(\mathrm{P}>0.05)$.

Serum vWF was positively associated with IR $(\mathrm{P}<0.05)$. No correlation was observed between vWF and W, BMI, WC, HC, WHR, TC, TG, LDL, HDL, FBG, FIN, SBP, and DBP (P>0.05).

Serum VE-cad was positively associated with W, BMI, WC, HC, WHR, TG, FBG, FIN, SBP, and IR $(\mathrm{P}<0.05)$. Meanwhile, it was negatively associated with HDL. However, no correlation was found between VE-cad and TC, LDL, and DBP $(\mathrm{P}>0.05)$.

Serum TM was positively associated with W, BMI, WC, HC, WHR, TG, FIN, and IR $(\mathrm{P}<0.05)$. It was also negatively associated with HDL. However, no correlation was found between TM and TC, LDL, FBG, SBP, or DBP $(\mathrm{P}>0.05)$.

Serum VEGF was positively associated with WC, HC, WHR, FBG, FIN, and IR (P< 0.05). No correlation was noted between VEGF and W, BMI, TC, TG, LDL, HDL, SBP, and DBP $(\mathrm{P}>0.05$, Table 2).

Table 2. Correlation analysis among PAI-1, vWF, VE-cad, TM and VEGF.

\begin{tabular}{|c|c|c|c|c|c|c|c|c|c|c|}
\hline \multirow[b]{2}{*}{ Group } & \multicolumn{2}{|c|}{ PAI-1 } & \multicolumn{2}{|c|}{ VWF } & \multicolumn{2}{|c|}{ VE-cad } & \multicolumn{2}{|c|}{ TM } & \multicolumn{2}{|c|}{ VEGF } \\
\hline & $r_{\mathrm{s}}$ & $P$ & $r_{\mathrm{s}}$ & $P$ & $r_{\mathrm{s}}$ & $\mathrm{P}$ & $r_{\mathrm{s}}$ & $\mathrm{P}$ & $r_{\mathrm{s}}$ & $P$ \\
\hline W & 0.489 & $0.006^{*}$ & 0.012 & 0.061 & 0.430 & $0.000^{*}$ & 0.247 & $0.019^{*}$ & 0.353 & 0.054 \\
\hline BMI & 0.534 & $0.002 *$ & 0.029 & 0.787 & 0.510 & $0.000 *$ & 0.379 & $0.000 *$ & 0.097 & 0.364 \\
\hline WC & 0.423 & $0.020 *$ & 0,151 & 0.158 & 0.522 & $0.000^{*}$ & 0.381 & $0.000 *$ & 0.235 & 0.027 * \\
\hline $\mathrm{HC}$ & 0.216 & 0.251 & 0.119 & 0.267 & 0.463 & $0.000 *$ & 0.333 & $0.001 *$ & 0.216 & $0.042 *$ \\
\hline WHR & 0.586 & $0.001^{*}$ & 0.158 & 0.140 & 0.481 & $0.001 *$ & 0.394 & $0.000 *$ & 0.211 & $0.048^{*}$ \\
\hline $\mathrm{TC}$ & 0.663 & $0.000 *$ & 0.116 & 0.295 & 0.101 & 0.341 & 0.107 & 0.316 & 0.038 & 0.735 \\
\hline TG & 0.645 & $0.000^{*}$ & 0.104 & 0.347 & 0.372 & $0.000 *$ & 0.248 & $0.019^{*}$ & 0.152 & 0.168 \\
\hline HDL & -0.533 & $0.002 *$ & 0.023 & 0.833 & -0.263 & $0.012 *$ & -2.245 & $0.020 *$ & -0.343 & 0.055 \\
\hline LDL & 0.419 & $0.021 *$ & 0.666 & 0.548 & 0.093 & 0.383 & 0.126 & 0.237 & 0.544 & 0.067 \\
\hline FBG & 0.419 & $0.021 *$ & 0.063 & 0.557 & 0.295 & $0.005^{*}$ & 0.177 & 0.096 & 0.243 & $0.030 *$ \\
\hline FINS & 0.405 & $0.026^{*}$ & 0.202 & 0.579 & 0.299 & $0.004 *$ & 0.298 & $0.004 *$ & 0.274 & $0.017^{*}$ \\
\hline SBP & 0.492 & $0.006^{*}$ & 0.029 & 0.079 & 0.213 & $0.044 *$ & 0.081 & 0.450 & 0.064 & 0.562 \\
\hline DBP & 0.409 & $0.025^{*}$ & 0.064 & 0.560 & 0.189 & 0.075 & 0.044 & 0.682 & 0.022 & 0.839 \\
\hline IR & 0.024 & 0.052 & 0.232 & $0.028^{*}$ & 0.315 & $0.003 *$ & 0.317 & $0.002 *$ & 0.302 & $0.004 *$ \\
\hline
\end{tabular}

$* \mathrm{P}<0.005$ indicates a positive correlation. $\mathrm{W}=$ weight; $\mathrm{BMI}=$ body mass index; $\mathrm{WC}=$ waist circumference; $\mathrm{HC}=$ hip circumference; WHR = waist/hip ratio; TC = serum total cholesterol; $\mathrm{TG}=$ serum triglycerides; $\mathrm{HDL}=$ highdensity lipoprotein; LDL = low-density lipoprotein; FBG = fasting blood glucose; FINS = fasting insulin; $\mathrm{SBP}=$ systolic blood pressure; DBP = diastolic blood pressure; IR = insulin resistance index. For other abbreviations, see legend to Table 1. 


\section{DISCUSSION}

The prevalence of overweight and obesity is increasing among Chinese children and adolescents, with a particularly rapid increase being observed in recent years (Ma, 2006). In 2007, on the basis of global diagnostic criteria for adults (Eckel et al., 2005), the IDF defined unified diagnostic criteria for children and adolescents with MS (Zimmet et al., 2007). However, the IDF adult criteria are still used in the definition of serum lipid levels and blood pressure and in the diagnosis of blood sugar abnormalities in order to achieve unified in diagnostic criteria in children and adults (Pi-Sunyer, 2000). This unified standard is of great significance for the prevention and treatment long-term outcomes including CVD and T2DM.

MS, being an aggregation of multiple cardiovascular risk factors, is closely associated with the occurrence and development of CVD. In recent years, a growing number of studies have shown that risk factors for adult atherosclerotic heart disease may stem from childhood (Deckelbaum and Williams, 2001; Steinberger and Daniels, 2003; Cook et al., 2003). The vascular endothelium (VEC) is an important part of the human body. The structure and function is important for the regulation of vasomotor functions, maintenance of vessel walls, anti-platelet aggregation, and endocrine functions. Its anatomical location is on a vulnerable functional interface, and various stimuli including injury and cardiovascular risk factors act upon it, which may lead to impaired function or disorders, thereby causing ECD (Pinkney et al., 1997). Many studies have shown that the ECD is an early pathophysiological indicator of CVD. ECD appears prior to cardiovascular disease, indicating the presence or imminent development of various cardiovascular risk factors. Early recognition and intervention are of extreme importance in the prevention, treatment, and prognosis of cardiovascular diseases in MS patients. The safeguarding of vascular endothelial function has become one of the goals of the treatment in adults with MS. The relationship between adult MS and ECD is already quite clear; however, whether these pathophysiological changes in the vascular endothelium also occur in children and adolescents with MS still needs to be investigated, as they can play a significant role in the prevention of adverse events in adult life.

A variety of substances secreted by the VEC, including PAI-1, vWF, VE-cad, TM, and VEGF, are seen as important indicators of endothelial cell function. PAI-1, currently used as a marker of ECD, is a single-chain glycoprotein that inhibits the activity of tissue-type plasminogen activator (t-PA) and urokinase-type plasminogen activator (u-PA). It can therefore decrease the fibrinolytic activity, and contribute to the formation of atherosclerotic plaques (Binder et al., 2002; Hua et al., 2002; Vaughan, 2005). vWF is a macromolecular plasma glycoprotein, in which may lead to increases in blood viscosity and risk of thrombotic disease. In addition, vWF can release platelet smooth muscle cell growth factor, and stimulate the migration and proliferation of vascular smooth muscle cells. On this basis, it can promote lipid deposition, exacerbate atherosclerosis, and worsen the original vascular thrombosis lesions (Ruggeri, 2001). VE-cad, the endothelial cell-specific cadherin that is specifically expressed on the surface of endothelial cells, is concentrated in the junctions of the endothelial cells (Martinez-Dolz et al., 2008). It plays an important role in the regulation of microvascular endothelial permeability. Detection of VE-cad expression can indirectly infer the existence of increasing vascular permeability and microvascular endothelial cell injury (Cheng et al., 2006).

As a glycoprotein with anticoagulant activity that exists on the endothelial cell surface, TM is an important anticoagulant cofactor (Orbe et al., 1995). As TM is present on the surface 
of endothelial cells, TM levels in normal plasma are low, but when vascular endothelial cells are injured, TM will leak into blood, thereby, significantly increasing plasma TM levels. Therefore, the plasma TM level can be used as a marker of endothelial injury (Laszik et al., 2001).

VEGF is a selective growth factor that contributed to angiogenesis by promoting mitotic division (Binder et et al., 2002). VEGF also enhances vascular permeability, thereby increasing exudation of blood components (Li et al., 1999). Growth factors, inflammatory cytokines, and hypoxia can stimulate the secretion of VEGF, and we therefore speculate that the accumulation of adipose tissue in obese children can cause the secretion of a variety of inflammatory cytokines, including interleukin-6 (IL-6), which may then stimulate increased VEGF secretion.

In this study, we verified the presence or absence of the ECD by determining serum levels of PAI-1, vWF, VE-cad, TM, and VEGF, and our results showed that the average serum levels of PAI-1, vWF, VE-cad, TM, and VEGF were significantly higher among children with MS than among the control group. This difference was statistically significant $(\mathrm{P}<0.05)$. Therefore, children with MS combined with ECD, PAI-1, vWF, VE-cadherin, TM, and VEGF can be used as predictive indicators for the identification of early vascular changes.

In this study, there was no statistically significant differences in serum vWF between the obese group and the control group $(\mathrm{P}=0.556)$, and serum PAI-1, VE- cad, TM, and VEGF levels in the obese group were significantly higher than those in the control group $(\mathrm{P}<0.05)$. We therefore suggest that pathophysiological vascular endothelial changes occur in MS patients as well as obese individuals without MS. Huang et al. (2010) also found, using B ultrasound detection of carotid intima-media thickness, that obese children and adolescents without MS had ECD. Therefore, the clinical prevention and control of ECD should not only focus solely on individuals with MS but also on individuals who are obese and do not have MS (Ji, 2007).

The most common clinical symptoms in children and adolescents with MS are as follows: obesity, especially abdominal obesity; dyslipidemia, mainly presented as increases in TG, TC, and LDL, and decreases in HDL; increase in BP; hyperinsulinemia; and T2DM (Carr et al., 2004). Currently, obesity (especially abdominal obesity) and insulin resistance are recognized as the most important pathogenic factors of MS (Nesto, 2003), and a direct relationship between the clinical and pathophysiological signs of MS and CVD has been reported (Iannuzzi et al., 2006). Through different mechanisms, each component of MS can negatively influence vascular endothelial function. ECD is mainly featured by increased vasoconstriction, a prothrombotic state, and smooth muscle cell proliferation. Accordingly, the potential mechanisms of MS damage on vascular endothelial function are as follows (Aggoun, 2007): 1) disturbance in carbohydrate metabolism (hyperinsulinemia/insulin resistance or hyperglycemia/diabetes), dyslipidemia, obesity, and hypertension can all cause vascular contractility and thereby increase diastolic dysfunction through a variety of channels; 2) under normal conditions, endothelial cells can prevent thrombosis due to their anticoagulant properties; 3 ) disturbance in carbohydrate metabolism, dyslipidemia, hypertension, and obesity can all increase PAI-1, vWF, VE-cadherin, TM, and VEGF production, leading to impaired anticoagulant and fibrinolytic activity, causing hypercoagulability, a low fibrinolytic and high viscosity state, promoting the formation of microthrombi, and thereby increasing the risk of cardiovascular disease; 4) when hyperglycemia, hyperlipemia, and hypertension occur, the release of various cytokines such as interleukin-1 (IL-1), IL-6, and platelet-derived growth factor (PDGF) are increased, which can promote the migration and proliferation of vascular smooth muscle cells, 
and also play an important role in the ECD process.

All of the components of MS are also cardiovascular risk factors. If various symptoms coexist, the effects may be more serious. In this study, correlation analysis showed that concentrations of PAI-1, vWF, VE-cad, TM, and VEGF are correlated with multiple factors including W, BMI, WC, HC, WHR, TC, TG, LDL, HDL, FBG, FIN, SBP, DBP, and IR. Thus, we suggest that the existence of ECD in children and adolescents with MS may be associated with obesity (especially abdominal obesity), increase in blood lipids and blood glucose, and higher BP. It has been reported that high blood sugar may cause monocytes to produce proinflammatory cytokines, increasing the production of IL-6, IL-1, and tumor necrosis factor (Wen et al., 2006), and that all these factors are precipitating factors for early vascular lesions. At the same time, in another study, no elevated proinflammatory cytokines were observed in patients with normal lipid levels and elevated blood sugar (Kouroedov et al., 2004). In addition, Kanter et al. (2007) have proposed that the process of hyperglycemia causing vascular endothelial lesions requires concomitant lipid activation and elevation and that it could enhance the damaging effects of blood glucose on vascular endothelial cells. Our results are consistent with these findings. We conclude that the presence of pathoglycemia and dyslipidemia in MS patients may be a predictive factor for vascular lesions.

\section{Conflicts of interest}

The authors declare no conflict of interest.

\section{ACKNOWLEDGMENTS}

We thank the anonymous reviewers for reviewing this manuscript.

\section{REFERENCES}

Aggoun Y (2007). Obesity, metabolic syndrome, and cardiovascular disease. Pediatr. Res. 61: 653-659.

Binder BR, Christ G, Gruber F, Grubic N, et al. (2002). Plasminogen activator inhibitor 1: physiological and pathophysiological roles. News Physiol. Sci. 17: 56-61.

Carr DB, Utzschneider KM, Hull RL, Kodama K, et al. (2004). Intra-abdominal fat is a major determinant of the National Cholesterol Education Program Adult Treatment Panel III criteria for the metabolic syndrome. Diabetes 53: 2087-2094.

Cheng WS, Zhang Z, Bai F and Huang Y (2006). Significance of soluble vascular endothelial cadherin in patients with coronary artery disease. China J. Modern Med. 16: 851-857.

Cook S, Weitzman M, Auinger P, Nguyen M, et al. (2003). Prevalence of a metabolic syndrome phenotype in adolescents: findings from the third National Health and Nutrition Examination Survey, 1988-1994. Arch. Pediatr. Adolesc. Med. 157: 821-827.

Deckelbaum RJ and Williams CL (2001). Childhood obesity: the health issue. Obes. Res. 9 (Suppl 4): 239S-243S.

Eckel RH, Grundy SM and Zimmet PZ (2005). The metabolic syndrome. Lancet 365: 1415-1428.

Expert Panel on Detection Evaluation aToHBCiA (2001). Executive Summary of The Third Report of The National Cholesterol Education Program (NCEP) Expert Panel on Detection, Evaluation, And Treatment of High Blood Cholesterol In Adults (Adult Treatment Panel III). JAMA 285: 2486-2497.

Hua Y, Xi G, Keep RF, Wu J, et al. (2002). Plasminogen activator inhibitor-1 induction after experimental intracerebral hemorrhage. J. Cereb. Blood Flow Metab. 22: 55-61.

Huang K, Zou CC, Yang XZ, Chen XQ, et al. (2010). Carotid intima-media thickness and serum endothelial marker levels in obese children with metabolic syndrome. Arch. Pediatr. Adolesc. Med. 164: 846-851.

Iannuzzi A, Licenziati MR, Acampora C, Renis M, et al. (2006). Carotid artery stiffness in obese children with the metabolic syndrome. Am. J. Cardiol. 97: 528-531. 
Ji CY (2007). The prevention and treatment of metabolic syndrome should start from children and adolescents. Chinese J. Child Health Care 15: 217-218.

Kanter JE, Johansson F, LeBoeuf RC and Bornfeldt KE (2007). Do glucose and lipids exert independent effects on atherosclerotic lesion initiation or progression to advanced plaques? Circ. Res. 100: 769-781.

Kouroedov A, Eto M, Joch H, Volpe M, et al. (2004). Selective inhibition of protein kinase Cbeta2 prevents acute effects of high glucose on vascular cell adhesion molecule-1 expression in human endothelial cells. Circulation 110: 91-96.

Laszik ZG, Zhou XJ, Ferrell GL, Silva FG, et al. (2001). Downregulation of endothelial expression of endothelial cell protein $\mathrm{C}$ receptor and thrombomodulin in coronary atherosclerosis. Am. J. Pathol. 159: 797-802.

Li X, Li ZB, Gao YQ, Yang P, et al. (1999). Expression of vascular endothelial growth factor and its gene in myocardium of spontaneously hypertensive rats. Chinese J. Pathophysiol. 15: 585.

Lim HS, Lip GY and Blann AD (2004). Plasma von Willebrand factor and the development of the metabolic syndrome in patients with hypertension. J. Clin. Endocrinol. Metab. 89: 5377-5381.

Ma J (2006). Metabolic syndrome in children. Chinese J. School Health 27: 829-831.

Martinez-Dolz L, Almenar L, Reganon E, Vila V, et al. (2008). Follow-up study on the utility of von Willebrand factor levels in the diagnosis of cardiac allograft vasculopathy. J. Heart Lung Transplant. 27: 760-766.

Nesto RW (2003). The relation of insulin resistance syndromes to risk of cardiovascular disease. Rev. Cardiovasc. Med. 4 (Suppl 6): S11-S18.

Orbe I, Paramo JA, Pinacho A, Hermida J, et al. (1995). Plasma thrombomodulin is increased in cord blood of healthy newborns. Thromb. Haemost. 73: 326.

Pi-Sunyer FX (2000). Obesity: criteria and classification. Proc. Nutr. Soc. 59: 505-509.

Pinkney JH, Stehouwer CD, Coppack SW and Yudkin JS (1997). Endothelial dysfunction: cause of the insulin resistance syndrome. Diabetes 46 (Suppl 2): S9-13.

Ruggeri ZM (2001). Structure of von Willebrand factor and its function in platelet adhesion and thrombus formation. Best. Pract. Res. Clin. Haematol. 14: 257-279.

Steinberger J and Daniels SR (2003). Obesity, insulin resistance, diabetes, and cardiovascular risk in children: an American Heart Association scientific statement from the Atherosclerosis, Hypertension, and Obesity in the Young Committee (Council on Cardiovascular Disease in the Young) and the Diabetes Committee (Council on Nutrition, Physical Activity, and Metabolism). Circulation 107: 1448-1453.

Vaughan DE (2005). PAI-1 and atherothrombosis. J. Thromb. Haemost. 3: 1879-1883.

Wen Y, Gu J, Li SL, Reddy MA, et al. (2006). Elevated glucose and diabetes promote interleukin-12 cytokine gene expression in mouse macrophages. Endocrinology 147: 2518-2525.

Zimmet P, Alberti G, Kaufman F, Tajima N, et al. (2007). The metabolic syndrome in children and adolescents. Lancet 369: 2059-2061. 